\title{
Pattern of Heart Valve Involvement in Rheumatic Heart Disease
}

\author{
Koju R, ${ }^{\star}$ Gurung R, ${ }^{\star}$ Pant P*, Pokharel B, ${ }^{\star}$ Bedi TRS ${ }^{\star}$ \\ ${ }^{*}$ Dhulikhel Hospital, Kathmandu University Hospital \\ Corresponding Author: Dr. Rajendra Koju, \\ Dhulikhel Hospital, Kathmandu University Hospital, Dhulikhel Nepal \\ Email: koju@mos.com.np
}

\begin{abstract}
Rheumatic heart disease is the most important consequence of acute rheumatic fever. Both are common cardiovascular problems in Nepal. Echocardiographic detection of rheumatic heart disease is important to establish the diagnosis. The involvement of valves and their severity guides the therapeutic options. A total of 133 valvular heart disease cases attended in Dhulikhel Hospital between July 2008 to June 2009 were analyzed. Fifty-one patients, in whom the problems were rheumatic in origin were studied. Among them, 12\% (6) had isolated aortic valve involvement, $35 \%(18)$ had isolated mitral valve and 53\%(27) ahd mixed involvement. Severe mitral stenosis accounts for $24 \%$ of all mitral stenosis and severe aortic stenosis is $20 \%$ fo all aortic stenosis. The rates for severe mitral regurgitation and severe aortic regurgitaiton are $30 \%$ and $28 \%$ respectively. Although the study population has a high number of female patients, the differences in the rates of involvement of aortic or mitral valve in both genders are statistically insignificant. The study, although small, confirms that in this population, females are more commonly affected, that the mitral valve is the most commonly damaged valve and that disease affecting multiple valves is marginally more common than isolated valve disease. The detection of valvular involvement at different stages can guide the therapeutic options.
\end{abstract}

Keywords : Rheumatic heart disease, rheumatic fever, echocardiography

\section{INTRODUCTION}

Rheumatic heart disease is the most important consequence of acute rheumatic fever. Rheumatic fever is non-suppurative complication of group A streptoccoccal pharyngitis due to a delayed immune response. Rheumatic fever usually occurs in childhood, affecting 5-15 year olds.

Rheumatic heart disease used to be a common cardiovascular problems of developed countries, which now continues to be a common health which now continues to be a common health problem in the developing world, causing morbidity and mortality among both children and adults. Rheumatic fever and rheumatic heart disease are common cardiovascular problems in Nepal.

Different school survey in Nepal reported the prevalence of rheumatic heart disease is high. Both rheumatic fever and rheumatic heart disease are more common in females.

However rheumatic heart disease can present in adults from aged 20 to 30 years. In $20 \%$ to $30 \%$ of such cases, there is no cardiac involvement, but patient often contract rheumatic fever more than once, and the damage is cumulative. Rheumatic heart valve lesion is progressive which lead to congestive cardiac failure.

Echocardiography is the important tool to detect rheumatic carditis and valvular morphology. 2D M-mode and doppler echocardiography can detect that valvular pathology, which is missed in clinical auscultation. It may over-diagnose trivial regurgitation because of its high snsitivity, and such trivial abnormalities, in the absence of any other abnormalities, can be disregarded.
The involvement of valves in rheumatic heart disease is very important from the therapeutic point of view. The treatment options depend upon the severity and type of valve involvement. This study aims to find out the pattern of involvement of heart valves and their severity.

\section{METHODOLOGY}

A total of 133 patients attending Dhulikhel Hospital with echocardiographically confirmed valvular heart disease between July 2008 to June 2009 were studied. Of this group 51 patients were having rheumatic heart disease. To be included in this group patients had either 1) a past history of rheumatic fever satisfying the revised Jones' criteria or 2) a pattern of morphological change on echocardiograpy consistent with a rheumatic heart disease causation once all other possible aetiologies had been excluded.

The transthoracic echocardiographic examination of the heart valves was performed with Toshiba Power Vision 6000 echocardiography machine. The grading of the stenosis was done with planimetry and pressure half time in mitral valve and peak pressure gradient in aortic valve. The grading of the valve regurgitation was done according to the regurgitant jet size and proximal jet width. Both stenosis and regurgitation was graded into Mild, Moderate or Severe.

The data was analyzed using SPSS 13.0.

@Nepalese Heart Journal. Nepalese Heart Journal retains copyright and work is simultaneously licensed under Creative Commons Attribution License CC - BY 4.0 that allows others to share the work with an acknowledgement of the work's authorship and initial publication in this journal. 


\begin{tabular}{l|l|l|l|}
\multirow{2}{*}{ Table 1: Age and Sex distribution of study population. } \\
\cline { 2 - 4 } Age group & \multicolumn{2}{|c}{ SEX } & \multirow{2}{*}{ Total } \\
\cline { 2 - 4 } & Male & Female & \\
\hline Less than 15 & 3 & 3 & 6 \\
\hline $16-30$ & 5 & 15 & 20 \\
\hline $31-45$ & 2 & 15 & 17 \\
\hline More than 46 & 2 & 6 & 8 \\
\hline Total & 12 & 39 & 51 \\
\hline
\end{tabular}

\begin{tabular}{|c|c|c|}
\hline \multirow{2}{*}{$\begin{array}{c}\text { Mitral } \\
\text { Stenosis }\end{array}$} & \multicolumn{2}{|c|}{ Sex } \\
\hline & Male & Female \\
\hline
\end{tabular}

\begin{tabular}{l|l|l|l} 
Mild & 3 & 7 & 10 \\
\hline Moderate & 0 & 9 & 9 \\
\hline Severe & 1 & 5 & 6 \\
\hline Total & 4 & 21 & 25 \\
\hline Total & 12 & 39 & 51 \\
\hline
\end{tabular}

\section{RESULT}

This study looked at all patients presenting to the cardiology department of Dhulikhel Hospital Over a period of a year.

A total of 51 patients having echocardiographically confirmed rheumatic heart disease were studied. Of this group $39(76.5 \%)$ were female and $12(23.5 \%)$ male. The mean age of study population was $32.82 \pm 15.33$ (standard deviation). The minimum age was 8 years and the maximum was 64 years. The majority of patients in this study were in the 16-30 age group.

\begin{tabular}{l|l|l|l}
\multirow{2}{*}{$\begin{array}{c}\text { Table 3. Mitral } \\
\text { Regurgitaion }\end{array}$} & \multicolumn{1}{|c}{ SEX } & \multicolumn{1}{c|}{ Total } & \multicolumn{1}{c}{ Percentage } \\
\cline { 2 - 4 } & Male & Female & 16.9 \\
\hline Mild & 3 & 3 & 6 \\
\hline Moderate & 6 & 14 & 20 \\
\hline Severe & 1 & 10 & 11 \\
\hline Total & 10 & 27 & 37 \\
\hline Total & 12 & 39 & 51 \\
\hline
\end{tabular}

The majority of patients had mitral valve involvement with regurgitation proving more common than stenosis. Mixed mitral valve disease and mixed mitral and aortic valve disease were found. Female patients accounted for $84 \%$ of mitral stenosis and $73 \%$ fo mitral regurgitation.

Aortic valve involvement was less common than mitral valve. Again females account for the greater number of cases with $66 \%$ for aortic stenosis and $71 \%$ for regurgitation. Isolated mitral valve involvement was 3 times more than aortic valve involvement. In $50 \%$ of cases mixed mitral and aortic valve involvement was demonstrated.

\begin{tabular}{|c|c|c|c|}
\hline \multirow{2}{*}{$\begin{array}{c}\text { Aortic } \\
\text { Stenosis }\end{array}$} & \multicolumn{2}{|c|}{ SEX } & \multirow{2}{*}{ Total } \\
\hline & Male & Female & \\
\hline Mild & 2 & 4 & 6 \\
\hline Moderate & 2 & 4 & 6 \\
\hline Severe & 1 & 2 & 3 \\
\hline Total & 5 & 10 & 15 \\
\hline Total & 12 & 39 & 51 \\
\hline
\end{tabular}

\begin{tabular}{l|l|l|l}
\multicolumn{2}{c}{ Table 5. Aortic Regurgitaiton } & \multirow{2}{*}{ Total } \\
\hline $\begin{array}{c}\text { Aortic } \\
\text { Regurgitaion }\end{array}$ & \multicolumn{2}{|c}{ SEX } \\
\cline { 2 - 4 } Mild & 2 & Female & \\
\hline Moderate & 4 & 14 & 5 \\
\hline Severe & 3 & 6 & 18 \\
\hline Total & 9 & 23 & 9 \\
\hline Total & 12 & 39 & 32 \\
\hline
\end{tabular}

In the study population 21 patients had secondary tricuspid regurgitaion graded from mild to severe. A pulmonary artery systolic pressure greater than $30 \mathrm{mmHg}$ (calculated from the tricuspid regurgitation velocity plus $10 \mathrm{mmHg}$ ) was shown in 19 patients. No patients had pulmonary valve involvement in this study.

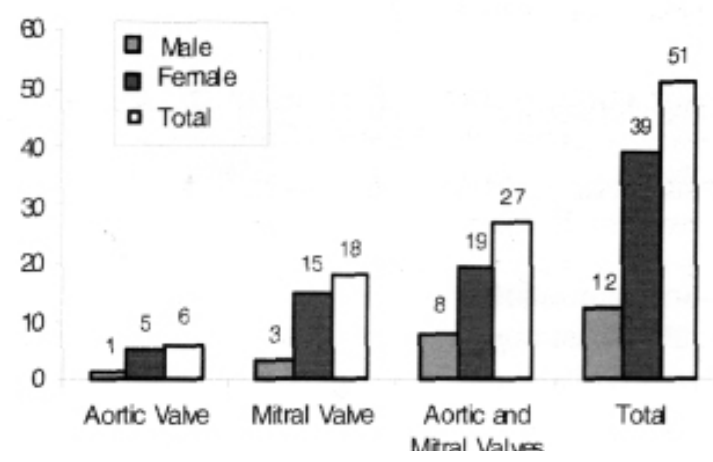

Figure 1: Involvement of values

Isolated aortic or mitral valve involvement rates are not significantly different $(p>0.05)$ in male and female. However, when all valve involvements are considered, females are more commonly affected.

\section{DIsCusSION}

The valvular abnormalities of rheumatic heart disease are the most important consequences of acute rheumatic fever and one of the commonest presentations of cardiovascular disease in hospitals in developing countries. The valvular lesions are progressive in nature and lead to congestive cardiac failure. Often rheumatic heart disease is asymptomatic and with no evidence of earlier acute rheumatic fever. In some cases rheumatic heart disease is diagnosed incidentally. 
Echocardiographic examination of rheumatic heart disease is important to detect and confirm the diagnosis, where clinical assessment is unreliable.

In the study patients with the valve abnormalities of rheumatic heart disease accounted for $38 \%$ of patients with valve disease. Female patients are more commonly affected. Rheumatic fever is more common in 5-15 years children. This study found more rheumatic heart disease among 16-45 years adults. The valvular involvement after rheumatic fever varies across geographical areas and depend upon socioeconomic status, rheumatic recurrence and severity of carditis.

\begin{tabular}{l|l|l|l}
\multirow{2}{*}{ Tabel 7. Pattern of mitral valve lesion } & \multirow{2}{*}{ SEX } \\
\cline { 2 - 3 } & Male & Female & \multirow{2}{*}{ Total } \\
\hline Mitral Stenosis & 2 & 4 & 6 \\
\hline Mitral Regurgitation & 1 & 5 & 6 \\
\hline Mitral Stenosis & 1 & 10 & 11 \\
\hline Regurgitaion & 6 & 6 & 6 \\
\hline Total & 3 & 15 & 18 \\
\hline
\end{tabular}

When rheumatic heart disease is present the mitral valve is most commonly affected. Isolated mitral valve involvement accounts for $35 \%$ of all rheumatic heart disease whereas the figure for isolated aortic valve is $12 \%$. The remaining $53 \%$ have mixed aortic and mitral valve involvement. Tricuspid regurgitation found in these patients is secondary to left sided valvular disease. The combination of valve involvement is often asymptomatic, with one valve having dominant dysfunction and other valve being less severely affected. Although the study population has high number of female patients, the differences in the rates of involvement aortic or mitral valve in both genders are statistically insignificant.

\begin{tabular}{l|l|l|l}
\multirow{2}{*}{ Table 8. pattern of aortic valve lesion } & \multirow{2}{*}{ SEX } \\
\cline { 2 - 3 } & Male & Female & \\
\hline Aortic Stenosis & 0 & 0 & 0 \\
\hline Aortic Regurgitation & 0 & 4 & 4 \\
\hline Aortic Stenosis & 1 & 10 & 11 \\
\hline Regurgitation & 1 & 1 & 2 \\
\hline Total & 1 & 5 & 6 \\
\hline
\end{tabular}

Severe mitral stenosis accounts for $24 \%$ of all mitral stenosis. Severe aortic stenosis is $20 \%$ of all aortic stenosis. The rates for severe mitral regurgitaion and severe aortic regurgitation are $30 \%$ and $28 \%$ respectively. Meira et.al. studied 258 children and adoloscents with acute rheumatic fever in Brazil and showed that $72.1 \%$ developed rheumatic valvular lesion. Among them $15.9 \%$ were severe. The severity depends upon the severity of carditis, mother's schooling, family income, rheumatic recurrences. The more severe patients found in out study could be due to the inclusion of already registered and treated cases.

In this study regurgitation was more common in patients with combined lesions of mitral and aortic valve than stenosis.

The study, although small, confirms that in this population females are more commonly affected. That the mitral valve is the most commonly damaged

\begin{tabular}{|c|c|c|c|}
\hline & \multicolumn{2}{|c|}{ SEX } & \multirow{2}{*}{ TOTAL } \\
\hline & Male & Female & \\
\hline MSAS & 0 & 1 & 1 \\
\hline MSAR & 0 & 2 & 2 \\
\hline ASMR & 000 & & \\
\hline ARMR & 3 & 3 & 0 \\
\hline MRASAR & 2 & 3 & 5 \\
\hline MS MRAR & 1 & 4 & 5 \\
\hline MS ASAR & 0 & 3 & 3 \\
\hline MS MR ASAR & 2 & 3 & 5 \\
\hline Total & 8 & 19 & 27 \\
\hline
\end{tabular}

Valve and that disease affecting multiple valves is marginally more common than isolated valve disease.

The detection of valvular involvement at different stages can guide the therapeutic options. Mild to moderate grades of valvular involvement without symptoms or minimal symptoms may be managed with secondary prophylaxis of rheumatic fever and other medical therapy. Severe grades of valvular involvement need surgical intervention-either repair or replacement.

I acknowledge the help from Dr. Alan Young who has commented helpfully on the manuscript of the article and Shilu Maskey for her technical assistance.

\section{REFERENCE}

1. Carapetis JR. Rheumatic heart disease in Asia Circulation 2008; 118: 2748-2753.

2. Carapetis JR, Steer AC, Mulholland EK, Weber M. The global burden of group A streptococcal disease. Lancet infect Dis 2005;5:685-694

3. Rheumatic fever and rheumatic heart disease. Report of a WHO study Group. World Health Organization, Geneva 2004 (Technical Report Series No 923)

4. the natural history of rheumatic fever and rheumatic heart disease: ten-year report of a cooperative clinical trial of ACTH, Cortisone, and Aspirin. A joint report by the rheumatic fever working party of the medical research council of Great Britain and the subcommittee of principal investigarors of the American council on rheumatic fever and congenital heart disease, American Heart Association. Circulation 1965; 32; 457-476.

5. Eisenberg MJ. Rheumatic heart disease in the developing world: prevalence, prevention and control. Eur Heart J. 1993;14;14;122-128

6. Shrestha UK, Bhattarai TN, Pandey MR. Prevalence of rheumatic fever and rheumatic heart disease in school children in a rural community of the hill region of Nepal. Indian Heart J 1991 Jan-Feb ;43(1);39-41

7. K.C. MB, Sharma D, shrestha MP, Rajbhandari S. 
Prevalence of rheumatic and congenital heart disease in school children on kathmandu valley in Nepal. Indian Heart J 2003; 55; 615-618

8. Regmi PR, pandey MR. Prevention of RF and RHD in school children of Kathmandu city, Indian Heat J 1997;49; 518-520

9. Gerber MA, Baltimore RS, Eaton CN, Gewitz M, Rowley AH, Shulman ST, Taubert KA. Prevention of Rheumatic Fever and Diagnosis and Treatment of Acute Streptococcal pharyngitis: A Scientific Statement from the American Heart Association Rheumatic Fever, Endocarditis, and kawasaki Disease Committee of the Council on Cardiovascular Disease in the young, the interdisciplinary Council on Functional Genomics and Translational Biology, and the Interdisciplicnary Council on Quality of Care and Outcomes Reseach: Endorsed by the American Academy of Pediatrics. Circulation 2009; 119; 1541-1551

10. Jones TD. Diagnosis of rheumatic fever journal of the American Medical Association 1944, 126:481-484

11. Ferrieri P. Proceedings of the Jones Criteria Workshop. Circulation 2002; 106; 2521-2523

12. Carapetis JR, Hardy M, Fakakovikaetau T. taib R, Wilkinson L, Penny DJ, Steer AC. Evaluation of a Screening Protocol Using Auscultation and Portable Echocardiography to Detect Asymptomatic Rheumatic Heart Disease in Tongan Schoolchildren. Nat Clin Pract Cardiovasc Med 2008;5(7):411-417.

13. Vasan RS, Shrivastava S, Vijayakumar, Narang R, Lister BC, Narula J. Echocardiographic Evaluation of Patients with Acute Rheumatic Fever and Rheumatic carditis. Circulation 1996;94:73-82.

14. Narula J, Chandrasekhar Y, Rahimtoola S. Diagnosis of active rheumatic carditis: the echoes of change Circulation 1999;100:1576-1581

15. Recommendations for evaluation of the severity of native valvular regurgitaiton with two dimensional and doppler echocardiography. American Society of Echocardiography 2004
16. Diagnosis and management of acute rheumatic fever/ rheumatic heart disease. Rheumatic heart disease program, World Heart Federation, reviewed and updated September 2007

17. Steinfeld L, Ritter S, Rappoport H, Martinez E. Silent rheumatic mitral regurgitation unmasked by Doppler studies Circulation 1986;74(suppl II):385.

18. www.medscape.com/viewarticle/574821 carapetis JR, hardy M, Fakakovikaetau T, Taib R, Wilkinson L, Penny DJ, Steer AC. Evaluation of a screening protocol using ausculatation and portable echocardiography to detect asymptomatic rheumatic heart disease in Tongan schoolchildren. Nature clinical Practice Cardiovascular Medicine published 07/01/2008

19. Folger GM Jr, Hajar R. Doppler echocardiographic findings of mitral and aortic valvular regurgitatiion in children manifesting only rheumatic arthritis. Am J cardiol. 1989;63:1278-1280.

20. Folger GM Jr, hajar R, Robida A, Hajar HA. Occurrence of valvar heart disease in acute rheumatic fever without evident carditis: colour-flow Doppler identification $\mathrm{Br}$ Heart J. 1992;67:434-439

21. Essop MR and Nkomo VT. Rheumatic and nonrheumatic valvular heart disease: Epidemiology, management, and prevention in Africa. Circulation 2005;112;3584-3591

22. Meira ZMA, Goulart EMA, Colosimo EA and Mota CCC. Longterm follow up of rheumatic fever and predictors of severe valval disease $\mathrm{n}$ Brazilian children and adolescents. Heart 2005;91:1019-1022

23. Shah PM. Tricuspid valve, pulmonary valve and multivalvular disease. Hurst't The Heart 12th edition 2008 by the McGraw-Hill Companies, Inc

24. 2008 Focused Update Incorporated into the ACC/AHA 2006 Guidelines for the Management of Patients with Valvular Heart Disease: A Report of the American College Disease: A Report of the American College of Cardiology/ American Heart Association task. Force on practice Guidelines. J. Am. Coll. Cardiol. 2008;52 (13);e1-e142 